\title{
Mycotic Abdominal Aortic Aneurysm Caused by Streptococcus equi
}

\author{
Danielle Schwartz $^{1}$, Donald McCarville ${ }^{2}$, Alexander Wong ${ }^{3}$
}

1. Department of Medicine, University of Saskatchewan, Saskatoon, CAN 2. Department of Vascular Surgery, University of Saskatchewan, Regina, CAN 3. Department of Infectious Diseases, University of Saskatchewan, Regina, CAN

Corresponding author: Danielle Schwartz, danielle.schwartz@usask.ca

\begin{abstract}
Streptococcus equi is a bacterium common in equine species and an uncommon pathogen in humans. Reported human infections can be severe and include meningitis, septic arthritis, and endocarditis. We report the case of a 64-year-old male who $S$. equi with several months of constitutional symptoms, back pain, and abdominal pain. Imaging demonstrated a large abdominal aortic aneurysm with a contained retroperitoneal rupture, with cultures from the aneurysm and blood cultures both positive for $S$. equi. The patient was successfully treated with open repair and placement of a Dacron graft and intravenous antibiotics and will remain on lifelong antibiotic prophylaxis.
\end{abstract}

Categories: Cardiac/Thoracic/Vascular Surgery, Internal Medicine, Infectious Disease Keywords: mycotic aneurysm, streptococcus equi, abdominal aortic aneurysm

\section{Introduction}

Mycotic aneurysms are arterial dilations caused by pathogens weakening the vascular wall. They are uncommon, accounting for $0.6 \%$ of aortic aneurysms [1], and have a good long-term prognosis with surgical repair. Staphylococcus aureus, Salmonella species, and Streptococcus species are the most common causative organisms [2]. The recommended therapy for mycotic aneurysms is surgical repair and at least six weeks of antibiotic therapy.

Streptococcus equi is a zoonotic pathogen that rarely causes infection in humans. There are three subspecies, S. equi subsp. equi, S. equi subsp. ruminatorum, and $S$. equi subsp. zooepidemicus, of which $S$. zooepidemicus is the subspecies reported to cause disease in humans. S. equi is a $\beta$-hemolytic Lancefield group C streptococcal bacterium and is the causative bacterium in $1.4 \%$ of all group C streptococcal infections [3]. While it most commonly causes infections in horses, it also causes infections in other animals including cats, pigs, and goats [4-7]. It can be transmitted to humans via contact with an infected animal or from the ingestion of unpasteurized dairy products [8]. S. equi usually enters the body through the respiratory and gastrointestinal tract, as well as through skin wounds [9]. It is closely related to Streptococcus pyogenes [8] and commonly causes similar sequelae of infection in humans such as glomerulonephritis [10], as well as serious infections including meningitis, septic arthritis, endocarditis, pneumonia, and osteomyelitis $[6,10-$

Review began 01/20/2021 Review ended 03/13/2021 Published 03/15/2021

\section{() Copyright 2021}

Schwartz et al. This is an open access article distributed under the terms of the Creative Commons Attribution License CC-BY 4.0., which permits unrestricted use, distribution, and reproduction in any medium, provided the original author and source are credited.
13]. Our report demonstrates that $S$. equi, a zoonotic Gram-positive coccus, can cause mycotic aneurysms, in addition to other invasive infections.

\section{Case Presentation}

A 64-year-old male presented to our facility with a three-month history of constitutional symptoms including generalized malaise, weight loss, anorexia, fatigue, and night sweats. Later in his course, but prior to presenting for medical care, he developed additional symptoms of back and abdominal pain. He was immunocompetent, and his medical history was significant for previous myocardial infarction, for which two drug-eluting stents were placed, and a perforated gastric ulcer repaired in 1990. He had no surgical history or family history of aortic aneurysms. His occupation was animal husbandry, and he worked on a farm that sells livestock with many cattle. He described an injury to the inside of his left leg after he slipped on ice and fell into cattle manure several months prior to the onset of his illness. It was a small open wound that was not reported to have clinical signs of infection; therefore, he did not receive antibiotics at that time. He did not smoke and denied past or current intravenous drug use.

As an outpatient, the patient previously underwent esophagogastroduodenoscopy to investigate his sixweek history of abdominal pain and was found to have a gastric ulcer positive for Helicobacter organisms. He was treated with triple therapy of clarithromycin, amoxicillin, and pantoprazole. All of his symptoms improved while on this regimen, but they relapsed thereafter.

Given his abdominal and back pain, a non-urgent computed tomography (CT) scan was performed three months after his symptoms developed, which demonstrated a large aneurysm in the infrarenal abdominal aorta measuring $8.2 \times 5.8 \times 8.5 \mathrm{~cm}$, with retroperitoneal fat stranding surrounding the aneurysm (Figures 
1A-1C). He had never had any previous imaging of his abdomen for comparison. The patient was immediately transferred to vascular surgery at our center for assessment and definitive management.

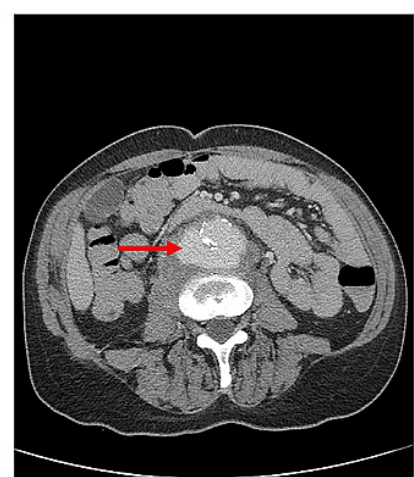

A.

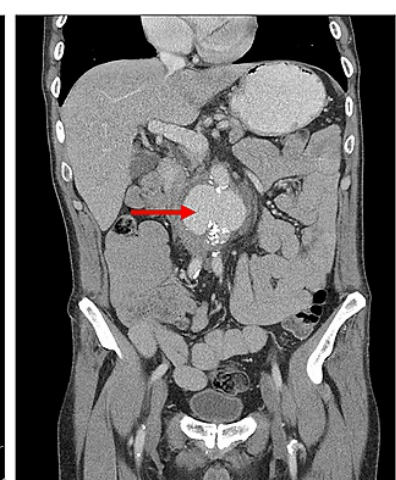

B.

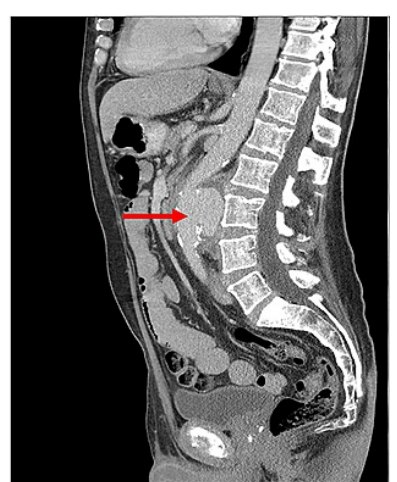

C.

FIGURE 1: (A) Axial view demonstrating circumferential calcification of the infrarenal abdominal aorta, with normal appearing diameter of aortic lumen, with associated posterior pseudoaneurysm. (B) Coronal view of infrarenal abdominal aorta with large posterior pseudoaneurysm. (C) Sagittal view, posterior pseudoaneurysm, with preserved aortic diameter above and below the arterial defect. Note the compression of the aortic lumen superior to the pseudoaneurysm.

On initial presentation to our facility, after the CT scan, the patient's physical exam demonstrated a tender pulsatile abdominal mass. He was afebrile and vital signs were within normal limits. Peripheral pulses were within normal limits. There were no clinical manifestations of infective endocarditis. Laboratory investigations showed white blood cell count 10.3 x 109/L, hemoglobin $94 \mathrm{~g} / \mathrm{L}$, platelets 433 x 109/L, with elevated inflammatory markers (ESR $105 \mathrm{~mm} / \mathrm{hr}, \mathrm{CRP} 95.6 \mathrm{mg} / \mathrm{L}$ ). The patient was taken immediately to the operating room for repair of what appeared to be a contained ruptured abdominal aortic aneurysm. Initial intra-operative intravenous antibiotics consisted of $2 \mathrm{~g}$ of cefazolin. Operative findings consisted of a small amount of ascites and a large abdominal aortic aneurysm with peri-aneurysmal edema, but without the expected peri-aneurysmal hematoma. After control and clamping of the vessels and upon opening the aorta, total loss of the posterior wall of the vessel was discovered, with a contained retroperitoneal rupture. The infected tissues were debrided and repair was completed with a synthetic Dacron prosthesis. Intra-operative samples were submitted including swabs of the presumed infected material as well as a piece of thrombus. Given the suspected mycotic nature of the aneurysm, post-operative antibiotics were broadened to piperacillin/tazobactam and vancomycin.

All intra-operative cultures as well as post-operative blood cultures were positive for $S$. equi, susceptible to penicillin (MIC $\leqslant 0.06$ ). The infectious disease service transitioned antibiotics to ceftriaxone $2 \mathrm{~g}$ IV q24h. Blood cultures drawn 96 hours post-operatively were sterile, and the patient's clinical condition improved rapidly. Following an unremarkable seven-day post-operative course, the patient was discharged home with a six-week course of ceftriaxone from the day of blood culture sterilization.

At the completion of his course of intravenous antibiotics, the patient reported near-complete resolution of symptoms with reassuring laboratory investigations. White blood cell count was 4.7 x 109/L, hemoglobin $100 \mathrm{~g} / \mathrm{L}$, platelets $323 \times 109 / \mathrm{L}$, and inflammatory markers were trending towards normal (ESR $64 \mathrm{~mm} / \mathrm{hr}$, CRP $14.4 \mathrm{mg} / \mathrm{L}$ ). Intravenous antibiotics were discontinued, and he began amoxicillin $500 \mathrm{mg}$ orally three times daily for six weeks. At review following 12 weeks of cumulative antibiotic therapy, he had returned to his previous functional baseline with no complaints of note, and laboratory investigations had normalized completely. He was switched to amoxicillin $500 \mathrm{mg}$ orally twice daily for lifelong prophylaxis.

A repeat CT scan of the aorta was performed four months after surgery, which showed mildly ectatic changes consisting of the Dacron prosthesis and peri-aortic tissues measuring $3.1 \times 3.9 \mathrm{~cm}$. No anostamotic pseudo-aneurysms were seen. A small intra-luminal linear density was noted but consisted of a localized fold in the Dacron graft, which is clinically insignificant.

\section{Discussion}

The patients with mycotic aneurysms tend to present with pain, fever, elevated inflammatory markers, leukocytosis and bacteremia, and many aneurysms are found ruptured when patients are taken for surgery 
$[14,15]$. Our patient presented with the majority of these symptoms but with insidious onset and months of non-specific symptoms, and incidental diagnosis following non-urgent imaging. The causative organism was found to be $S$. equi, which is not a typical organism that causes mycotic aneurysms. In our case, the subspecies of $S$. equi was not identified. The patient's risk factors were working on a farm with animals and his skin wound.

Our patient was treated with guideline-recommended therapy for mycotic aneurysms, which is surgical repair and at least six weeks of antibiotic therapy. Surgical approaches include in-situ repair with synthetic prosthesis such as with our patient, in-situ repair with autogenous tissue, or ligation and extra-anatomic bypass. The duration of antibiotic treatment ranges from six weeks to lifelong prophylaxis, depending on the causative organism and aneurysm location [16]. Aortic graft infections occur in 1\%-5\% of cases and are associated with significant morbidity and mortality, with a mortality rate of about $19 \%$ [17], and S. equi often causes severe infections. Therefore, our intent is for the patient to remain on lifelong antibiotic prophylaxis. $S$. equi has been reported to cause secondary infections of atherosclerotic abdominal aorta aneurysms and vascular grafts $[18,19]$. Our report demonstrates that the organism can cause mycotic aneurysms as well.

\section{Conclusions}

$S$. equi is an uncommon zoonotic bacterium that can cause a wide range of infections, including mycotic aneurysms. It should be considered when a patient presents with appropriate risk factors such as working closely with animals. Aneurysms caused by $S$. equi can be successfully treated in the same way as other bacterial mycotic aneurysms, with surgery to repair the aneurysm and prolonged antibiotic therapy, and lifelong prophylaxis in selected candidates.

\section{Additional Information \\ Disclosures}

Human subjects: Consent was obtained or waived by all participants in this study. Conflicts of interest: In compliance with the ICMJE uniform disclosure form, all authors declare the following: Payment/services info: All authors have declared that no financial support was received from any organization for the submitted work. Financial relationships: All authors have declared that they have no financial relationships at present or within the previous three years with any organizations that might have an interest in the submitted work. Other relationships: All authors have declared that there are no other relationships or activities that could appear to have influenced the submitted work.

\section{References}

1. Reddy DJ, Shepard AD, Evans JR, Wright DJ, Smith RF, Ernst CB: Management of infected aortoiliac aneurysms. Arch Surg. 1991, 126:873-879. 10.1001/archsurg.1991.01410310083012

2. Gross G, Harringer W, Mair R, Wimmer-Greinecker G, Klima U, Brücke P: Mycotic aneurysms of the thoracic aorta. Eur J Cardiothorac Surg. 1994, 8:135-138.

3. Barnham M, Kerby J, Chander R, Miller M: Group C streptococci in human infection: a study of 308 isolates with clinical correlations. Epidemiol Infect. 1989, 120:379-390. 10.1017/s0950268800030090

4. Timoney JF: The pathogenic equine streptococci. Vet Res. 2004, 35:397-409. 10.1051/vetres:2004025

5. Blum S, Elad D, Zukin N, et al.: Outbreak of Streptococcus equi subsp. Zooepidemicus infections in cats . Vet Microbiol. 2010, 144:236-239. 10.1016/j.vetmic.2009.12.040

6. Edwards AT, Roulson M, Ironside MJ: A milk-borne outbreak of serious infection due to Streptococcus zooepidemicus (Lancefield Group C). Epidemiol Infect. 2009, 101:43-51. 10.1017/s0950268800029204

7. Pisoni G, Zadoks RN, Vimercati C, Locatelli C, Zanoni MG, Moroni P: Epidemiological investigation of Streptococcus equi subspecies zooepidemicus involved in clinical mastitis in dairy goats. J Dairy Sci. 2009, 92:943-951. 10.3168/jds.2008-1548

8. Holden MTG, Heather Z, Paillot R, et al.: Genomic evidence for the evolution of Streptococcus equi: host restriction, increased virulence, and genetic exchange with human pathogens. PloS Pathog. 2009, 5:e1000346. 10.1371/journal.ppat.1000346

9. Bradley SF, Gordon JJ, Baumgartner DD, Marasco WA, Kauffman CA: Group C streptococcal bacteremia: analysis of 88 cases. Rev Infect Dis. 1991, 12:270-280. 10.1093/clinids/13.2.270

10. Barnham M, Ljunggren A, McIntyre M: Human infection with Streptococcus zooepidemicus (Lancefield group C): three case reports. Epidemiol Infect. 2009, 98:183-190. 10.1017/s0950268800061896

11. Eyre DW, Kenkre JS, Bowler ICJW, McBride SJ: Streptococcus equi subspecies zooepidemicus meningitis - a case report and review of the literature. Euro J Clin Microbiol. 2010, 29:1459-1463. 10.1007/s10096-010$1037-5$

12. Friederichs J, Hungerer S, Werle R, Militz M, Bühren V: Human bacterial arthritis caused by Streptococcus zooepidemicus: report of a case. Int J Infect Dis. 2010, 14:233-235. 10.1016/j.ijid.2009.08.009

13. Barson, W: Group C Streptococcal osteomyelitis. J Pediatr Orthoped. 1986, 6:346-348. 10.1097/01241398198605000-00016

14. Oderich GS, Panneton JM, Bower TC, et al.: Infected aortic aneurysms: aggressive presentation, complicated early outcome, but durable results. J Vasc Surg. 2001, 34:900-908. 10.1067/mva.2001.118084

15. Müller TM, Wegener OR, Grabitz K, Pillny M, Thomas L, Sandmann W: Mycotic aneurysms of the thoracic and abdominal aorta and iliac arteries: experience with anatomic and extra-anatomic repair in 33 cases. J Vasc Surg. 2011, 33:106-113. 10.1067/mva.2001.110356

16. Brown SL, Busuttil RW, Baker LD, Machleder HI, Moore SM, Barker WF：Bacteriologic and surgical 


\section{Cureus}

determinant of survival in patients with mycotic aneurysms. J Vasc Surg. 1984, 1:541-547. 10.1016/07415214(84)90040-5

17. Wilson WR, Bower TC, Creager MA, et al.: Vascular graft infections, mycotic aneurysms, and endovascular infections: a scientific statement from the American Heart Association. Circulation. 2016, 134:e412-460. 10.1161/CIR.0000000000000457

18. Albarracin C, Rosencrance G, Boland J, Hernandez JE: Bacteremia due to Streptococcus zooepidemicus associated with an abdominal aortic aneurysm. W V Med J. 1998, 94:90-92.

19. Altreuther M, Lange C, Myhre HO, Hannula R: Aortic graft infection and mycotic aneurysm with Streptococcus equi zooepidemicus: two cases with favorable outcome of antibiotic treatment. Vascular. 2012, $21: 6-9.10 .1258 /$ vasc.2011.cr0299 